This copy is for your personal, non-commercial use only.

If you wish to distribute this article to others, you can order high-quality copies for your colleagues, clients, or customers by clicking here.

Permission to republish or repurpose articles or portions of articles can be obtained by following the guidelines here.

The following resources related to this article are available online at www.sciencemag.org (this information is current as of April 17, 2013 ):

Updated information and services, including high-resolution figures, can be found in the online version of this article at:

http://www.sciencemag.org/content/338/6111/1190.full.html

Supporting Online Material can be found at:

http://www.sciencemag.org/content/suppl/2012/10/31/science.1227160.DC1.html

A list of selected additional articles on the Science Web sites related to this article can be found at:

http://www.sciencemag.org/content/338/6111/1190.full.html\#related

This article cites 56 articles, 3 of which can be accessed free:

http://www.sciencemag.org/content/338/6111/1190.full.htm|\#ref-list-1

This article has been cited by 1 articles hosted by HighWire Press; see:

http://www.sciencemag.org/content/338/6111/1190.full.html\#related-urls

This article appears in the following subject collections:

Astronomy

http://www.sciencemag.org/cgi/collection/astronomy 


\section{The Imprint of the Extragalactic Background Light in the Gamma-Ray Spectra of Blazars}

M. Ackermann, ${ }^{1}$ M. Ajello, ${ }^{2,3 *}$ A. Allafort, ${ }^{2}$ P. Schady, ${ }^{4}$ L. Baldini, ${ }^{5}$ ]. Ballet, ${ }^{6}$ G. Barbiellini, ${ }^{7,8}$ D. Bastieri, ${ }^{9,10}$ R. Bellazzini, ${ }^{11}$ R. D. Blandford, ${ }^{2}$ E. D. Bloom, ${ }^{2}$ A. W. Borgland, ${ }^{2}$ E. Bottacini, ${ }^{2}$ A. Bouvier, ${ }^{12}$ ]. Bregeon, ${ }^{11}$ M. Brigida, ${ }^{13,14}$ P. Bruel, ${ }^{15}$ R. Buehler, ${ }^{2 *}$ S. Buson, ${ }^{9,10}$ G. A. Caliandro, ${ }^{16}$ R. A. Cameron, ${ }^{2}$ P. A. Caraveo, ${ }^{17}$ E. Cavazzuti, ${ }^{18}$ C. Cecchi, ${ }^{19,20}$ E. Charles, ${ }^{2}$ R. C. G. Chaves, ${ }^{6}$ A. Chekhtman, ${ }^{21} \dagger$ C. C. Cheung, ${ }^{22} \dagger$ J. Chiang, ${ }^{2}$ G. Chiaro, ${ }^{23}$ S. Ciprini ${ }^{24,20}$ R. Claus, ${ }^{2}$ J. Cohen-Tanugi, ${ }^{25}$ ]. Conrad, ${ }^{26,27,28}$ S. Cutini, ${ }^{18}$ F. D'Ammando, ${ }^{19,29,30}$ F. de Palma, ${ }^{13,14}$ C. D. Dermer, ${ }^{31}$ S. W. Digel, ${ }^{2}$ E. do Couto e Silva, ${ }^{2}$ A. Domínguez, ${ }^{12}$ P. S. Drell, ${ }^{2}$ A. Drlica-Wagner, ${ }^{2}$ C. Favuzzi, ${ }^{13,14}$ S. J. Fegan, ${ }^{15}$ W. B. Focke, ${ }^{2}$ A. Franckowiak, ${ }^{2}$ Y. Fukazawa, ${ }^{32}$ S. Funk, ${ }^{2}$ P. Fusco, ${ }^{13,14}$ F. Gargano, ${ }^{14}$ D. Gasparrini, ${ }^{18}$ N. Gehrels, ${ }^{33}$ S. Germani, ${ }^{19,20}$ N. Giglietto, ${ }^{13,14}$ F. Giordano, ${ }^{13,14}$ M. Giroletti, ${ }^{34}$ T. Glanzman, ${ }^{2}$ G. Godfrey, ${ }^{2}$ I. A. Grenier, ${ }^{6}$ ]. E. Grove, ${ }^{31}{ }^{3}$. Guiriec, ${ }^{33}$ M. Gustafsson, ${ }^{9}$ D. Hadasch, ${ }^{16}$ M. Hayashida, ${ }^{2,35}$ E. Hays, ${ }^{33}$ M. S. Jackson, ${ }^{36,27}$ T. Jogler, $^{2}$ J. Kataoka, $^{37}$ J. Knödlseder, $^{38,39}$ M. Kuss, ${ }^{11}$ ]. Lande, ${ }^{2}$ S. Larsson, ${ }^{26,27,40}$ L. Latronico, ${ }^{41}$ F. Longo, ${ }^{7,8}$ F. Loparco, ${ }^{13,14}$ M. N. Lovellette, ${ }^{31}$ P. Lubrano, ${ }^{19,20}$ M. N. Mazziotta, ${ }^{14}$ J. E. McEnery, ${ }^{33,42}$ ]. Mehault, ${ }^{25}$ P. F. Michelson, ${ }^{2}$ T. Mizuno, ${ }^{43}$ C. Monte, ${ }^{13,14}$ M. E. Monzani, ${ }^{2}$ A. Morselli, ${ }^{44}$ I. V. Moskalenko, ${ }^{2}$ S. Murgia, ${ }^{2}$ A. Tramacere, ${ }^{45}$ E. Nuss, ${ }^{25}$ J. Greiner, ${ }^{4}$ M. Ohno, ${ }^{46}$ T. Ohsugi, ${ }^{43}$ N. Omodei, ${ }^{2}$ M. Orienti, ${ }^{34}$ E. Orlando, ${ }^{2}$ J. F. Ormes, ${ }^{47}$ D. Paneque, ${ }^{48,2}$ J. S. Perkins, ${ }^{33,49,50,51}$ M. Pesce-Rollins, ${ }^{11}$ F. Piron, ${ }^{25}$ G. Pivato, ${ }^{10}$ T. A. Porter, ${ }^{2,12}$ S. Rainò, ${ }^{13,14}$ R. Rando, ${ }^{9,10}$ M. Razzano, ${ }^{11,12}$ S. Razzaque, ${ }^{21} \dagger$ A. Reimer, ${ }^{52,2 *}$ O. Reimer, ${ }^{52,2}$ L. C. Reyes, ${ }^{53}$ S. Ritz, ${ }^{12}$ A. Rau, ${ }^{4}$ C. Romoli, ${ }^{10}$ M. Roth, ${ }^{54}$ M. Sánchez-Conde, ${ }^{2}$ D. A. Sanchez, ${ }^{55}$ J. D. Scargle, ${ }^{56}$ C. Sgrò, ${ }^{11}$ E. J. Siskind, ${ }^{57}$ G. Spandre, ${ }^{11}$ P. Spinelli, ${ }^{13,14}$ Łukasz Stawarz, $^{46,58}$ D. J. Suson, ${ }^{59}$ H. Takahashi, ${ }^{32}$ T. Tanaka, ${ }^{2}$ J. G. Thayer, ${ }^{2}$ D. J. Thompson, ${ }^{33}$ L. Tibaldo, ${ }^{9,10}$ M. Tinivella, ${ }^{11}$ D. F. Torres, ${ }^{16,60}$ G. Tosti, ${ }^{19,20}$ E. Troja, ${ }^{33}$ T. L. Usher, ${ }^{2}$ ]. Vandenbroucke, ${ }^{2}$ V. Vasileiou, ${ }^{25}$ G. Vianello, $^{2,61}$ V. Vitale, ${ }^{4,62}$ A. P. Waite, ${ }^{2}$ B. L. Winer, ${ }^{63}$ K. S. Wood, $^{31}$ M. Wood $^{2}$

The light emitted by stars and accreting compact objects through the history of the universe is encoded in the intensity of the extragalactic background light (EBL). Knowledge of the EBL is important to understand the nature of star formation and galaxy evolution, but direct measurements of the EBL are limited by galactic and other foreground emissions. Here, we report an absorption feature seen in the combined spectra of a sample of gamma-ray blazars out to a redshift of $z \sim 1.6$. This feature is caused by attenuation of gamma rays by the EBL at optical to ultraviolet frequencies and allowed us to measure the EBL flux density in this frequency band.

$\mathrm{T}$ he bulk of the intergalactic gas in the universe must have been reionized between the epoch of cosmic recombination, when the universe was only 300,000 years old $(z \sim 1100)$, and 1 billion years later $(z \sim 6)$, as indicated observationally by the spectra of distant quasistellar objects (1). However, the sources, modes, and nature of this cosmic reionization are largely unknown because most of this redshift range has yet to be explored. Photoionization by ultraviolet (UV) radiation, produced by the first stars and galaxies of the universe, represents the primary suspect for the ionizing process $(2,3)$. Direct detection of the UV radiation fields is thus of fundamental importance, but at present is extremely difficult (3).

An indirect but powerful means of probing the diffuse radiation fields is through $\gamma-\gamma$ absorption of high-energy gamma rays (4-6). In this process, a gamma-ray photon of energy $E_{\gamma}$ and an extragalactic background light (EBL) photon of energy $E_{\mathrm{EBL}}$ annihilate and create an electronpositron pair. This process occurs for head-on collisions when, for example, $E_{\gamma} \times E_{\mathrm{EBL}} \geq 2\left(m_{\mathrm{e}} c^{2}\right)^{2}$, where $m_{\mathrm{e}} c^{2}$ is the rest mass energy of the elec- tron. This introduces an attenuation in the spectra of gamma-ray sources above a critical gammaray energy of $E_{\text {crit }}(z) \approx 170(1+z)^{-2.38} \mathrm{GeV}(7,8)$.

The detection of the gamma-ray horizon (i.e., the point beyond which the emission of gammaray sources is strongly attenuated) is one of the primary scientific drivers of the Fermi GammaRay Space Telescope (9-11). Several attempts have been made in the past, but none detected the long-sought EBL attenuation (12-14). So far, limits on the EBL density have been inferred from the absence of absorption features in the spectra of individual blazars $(13,15)$, distant galaxies with bright gamma-ray emission powered by matter accreting onto central, massive black holes. Although this feature is indeed difficult to constrain for a single source, we show that it is detected collectively in the gamma-ray spectra of a sample of blazars as a cutoff that changes amplitude and energy with redshift. We searched for an attenuation of the spectra of blazars in the 1 to vations of the Large Area Telescope (LAT) on board the Fermi satellite. At these energies, gamma rays $500 \mathrm{GeV}$ band using the first 46 months of obser- are absorbed by EBL photons in the optical to UV range. Thanks to the large energy and redshift coverage, Fermi-LAT measures the intrinsic (i.e., unabsorbed) spectrum up to $\sim 100 \mathrm{GeV}$ for any blazar at $z<0.2$ and up to $\sim 15 \mathrm{GeV}$ for any redshift.

The LAT has detected $>1000$ blazars to date (16). We restricted our search to a subset of 150 blazars of the BL Lacertae (BL Lac) type that are significantly detected above $3 \mathrm{GeV}$ because of the expected lack of intrinsic absorption (17). The sample covers a redshift range of 0.03 to $1.6(18,19)$. The critical energy is therefore always $\geq 25 \mathrm{GeV}$, which means that the spectrum measured below this energy is unabsorbed and a true representation of the intrinsic spectrum of the source. We thus determined the intrinsic source spectrum relying on data between $1 \mathrm{GeV}$ and the critical energy $E_{\text {crit }}$ and extrapolated it to higher energies. By combining all the spectra, we were able to determine the average deviation, above the critical energy, of the measured spectra from the intrinsic ones, which ultimately provides a measurement of the optical depth $\tau_{\gamma \gamma}$.

The analysis was performed using the Fermi Science Tools (20). We determined the spectral parameters of each blazar by maximizing the likelihood of a given source model. The model comprised the Galactic and isotropic diffuse components and all sources in the second Fermi LAT catalog (21) within a region of interest (ROI) of $15^{\circ}$ radius. We modeled the spectra of the sources in our sample as parabolic in the logarithmic space of energy and flux [see equation 2 in (21) for a definition]. Their spectra were modified by a term $e_{\gamma \gamma}^{-\tau}{ }^{(E, z)}$ that describes the absorption of gamma-ray photons on the EBL. In the above, we defined $\tau_{\gamma \gamma}(E, z)=b \times \tau^{\text {model }}{ }_{\gamma \gamma}(E, z)$, where the $\tau_{\gamma \gamma}^{\text {model }}(E, z)$ is the optical depth predicted by EBL models $(7,22-25)$ and $b$ is a scaling variable, left free in the likelihood maximization. In particular, this allowed us to assess the likelihood of two important scenarios: (i) there is no EBL attenuation $(b=0)$, or (ii) the model prediction is correct $(b=1)$.

We combined the data from all the ROIs in a global fit that determined the common parameter $b$ for a given EBL model (see table S1). All those models with a minimal EBL density based on (or compatible with) resolved galaxy counts $(2,7,24-27)$ were found to be acceptable descriptions of the Fermi data (i.e., are consistent with $b=1$ within $\approx$ $25 \%$ ) (see also Fig. 1), yielding a significance of the absorption feature of up to $\sim 6 \mathrm{SD}$. Models that predict a larger intensity of the EBL, particularly in the UV $(22,23)$, would produce a stronger-thanobserved attenuation feature and are therefore incompatible with the Fermi observations. Our measurement points to a minimal level of the opticalUV EBL up to redshift $z \approx 1.6$, which combined with the upper limits $(15,28,29)$ derived at lower redshift (using observations of blazars at $\mathrm{TeV}$ energies) on the near-infrared EBL highlights the conclusion that most of the EBL intensity can be explained by the measured galaxy emission.

Our measurement relies on the accuracy of the extrapolation of the intrinsic spectra of the 
sources above the critical energy (30). This in turn depends on a precise description of the gamma-ray spectra by our source parametrization. To verify that this is the case and to exclude the possibility that the detected absorption feature is intrinsic to the gamma-ray sources (17), we performed the analysis in three independent redshift intervals $(z<0.2,0.2 \leq z<0.5$, and $0.5 \leq$ $z<1.6)$. The deviations from the intrinsic spectra in the three redshift intervals are displayed in Fig. 2. In the local universe $(z<0.2)$, EBL absorption is negligible in most of the Fermi-LAT energy band $\left(E_{\text {crit }} \geq 120 \mathrm{GeV}\right)$. The lowest redshift interval therefore reveals directly the intrinsic spectra of the sources and shows that our spectral parametrization is accurate (18). The absorption feature is clearly visible above the critical energy in the higher redshift bins. Its amplitude and modulation in energy evolve with redshift as expected for EBL absorption. In principle, the observed attenuation could be due to a spectral cutoff that is intrinsic to the gamma-ray sources. The absence of a cutoff in the spectra of sources with $z<0.2$ would require that the properties of BL Lacs change with redshift or luminosity. It remains an issue of debate whether such evolution exists (31-34). However, in case it were present, the intrinsic cutoff would be expected to evolve differently with redshift than we observe. To illustrate this effect, we fitted the blazar sample assuming that all the sources have an exponential cutoff at an energy $E_{0}$. From source to source, the observed cutoff energy changes because of the source redshift and because we assumed that blazars as a population are distributed in a sequence such as that proposed in (31-34). $E_{0}$ was fitted to the data globally like $b$ above. As
${ }^{1}$ Deutsches Elektronen Synchrotron DESY, D-15738 Zeuthen, Germany. ${ }^{2}$ W. W. Hansen Experimental Physics Laboratory, Kavli Institute for Particle Astrophysics and Cosmology, Department of Physics and SLAC National Accelerator Laboratory, Stanford University, Stanford, CA 94305, USA. ${ }^{3}$ Space Sciences Laboratory, 7 Gauss Way, University of California, Berkeley, CA 94720-7450, USA. ${ }^{4}$ Max-Planck Institut für Extraterrestrische Physik, 85748 Garching, Germany. ${ }^{5}$ Università di Pisa and Istituto Nazionale di Fisica Nucleare, Sezione di Pisa I-56127 Pisa, Italy. ${ }^{6}$ Laboratoire AIM, CEA-IRFU/CNRS/Université Paris Diderot, Service d'Astrophysique, CEA Saclay, 91191 Gif sur Yvette, France. ${ }^{7}$ Istituto Nazionale di Fisica Nucleare, Sezione di Trieste, I-34127 Trieste, Italy. ${ }^{8}$ Dipartimento di Fisica, Università di Trieste, I-34127 Trieste, Italy. ${ }^{9}$ Istituto Nazionale di Fisica Nucleare, Sezione di Padova, I-35131 Padova, Italy. ${ }^{10}$ Dipartimento di Fisica e Astronomia "G. Galilei," Università di Padova, I-35131 Padova, Italy. ${ }^{11}$ Istituto Nazionale di Fisica Nucleare, Sezione di Pisa, I-56127 Pisa, Italy. ${ }^{12}$ Santa Cruz Institute for Particle Physics, Department of Physics and Department of Astronomy and Astrophysics, University of California at Santa Cruz, Santa Cruz, CA 95064, USA. ${ }^{13}$ Dipartimento di Fisica "M. Merlin" dell'Università e del Politecnico di Bari, I-70126 Bari, Italy. ${ }^{14}$ Istituto Nazionale di Fisica Nucleare, Sezione di Bari, 70126 Bari, Italy. ${ }^{15}$ Laboratoire Leprince-Ringuet, École Polytechnique, CNRS/IN2P3, Palaiseau, France. ${ }^{16}$ Institut de Ciències de I'Espai (IEEE-CSIC), Campus UAB, 08193 Barcelona, Spain. ${ }^{17}$ INAF-Istituto di Astrofisica Spaziale e Fisica Cosmica, I-20133 Milano, Italy. ${ }^{18}$ Agenzia Spaziale Italiana (ASI) Science Data Center, I-00044 Frascati (Roma), Italy. ${ }^{19}$ Istituto Nazionale di Fisica Nucleare, Sezione di Perugia, I-06123 Perugia, Italy. ${ }^{20}$ Dipartimento di Fisica, Università degli Studi di Perugia, I-06123 Perugia, Italy. ${ }^{21}$ Center for Earth Observing and Space Research, College of Science, George Mason University, Fairfax, VA 22030, USA. ${ }^{22}$ National Re-

Fig. 1. Measurement, at the 68 and $95 \%$ confidence levels (including systematic uncertainties added in quadrature), of the opacity $\tau_{\gamma \gamma}$ from the best fits to the Fermi data compared with predictions of EBL models. The plot shows the measurement at $z \approx 1$, which is the average redshift of the most constraining redshift interval (i.e., $0.5 \leq z<$ 1.6). The Fermi-LAT measurement was derived combining the limits on the best-fit EBL models. The downward arrow represents the $95 \%$ upper limit on the opacity at $z=1.05$ derived in (13). For clarity, this figure shows only a selection of the models we tested; the full list is reported in table S1. The EBL models of (49), which are not defined for $E \geq 250$ / $(1+z) \mathrm{GeV}$ and thus could not be used, are reported here for completeness. search Council, National Academy of Sciences, Washington, DC 20001. ${ }^{23}$ INFN and Dipartimento di Fisica e Astronomia "G. Galilei," Università di Padova, I-35131 Padova, Italy. ${ }^{24}$ ASI Science Data Center, I-00044 Frascati (Roma), Italy. ${ }^{25}$ Laboratoire Univers et Particules de Montpellier, Université Montpellier 2, CNRS/IN2P3, Montpellier, France. ${ }^{26}$ Department of Physics, Stockholm University, AlbaNova, SE-106 91 Stockholm, Sweden. ${ }^{27}$ The Oskar Klein Centre for Cosmoparticle Physics, AlbaNova, SE-106 91 Stockholm, Sweden. ${ }^{28}$ Royal Swedish Academy of Sciences Research Fellow, SE-106 91 Stockholm, Sweden. ${ }^{29}$ IASF Palermo, 90146 Palermo, Italy. ${ }^{30}$ INAF-Istituto di Astrofisica Spaziale e Fisica Cosmica, I-00133 Roma, Italy. ${ }^{31}$ Space Science Division, Naval Research Laboratory, Washington, DC 20375-5352, USA. ${ }^{32}$ Department of Physical Sciences, Hiroshima University, Higashi-Hiroshima, Hiroshima 739-8526, Japan. ${ }^{33}$ NASA Goddard Space Flight Center, Greenbelt, MD 20771, USA. ${ }^{34}$ INAF Istituto di Radioastronomia, 40129 Bologna, Italy. ${ }^{35}$ Department of Astronomy, Graduate School of Science, Kyoto University, Sakyo-ku, Kyoto 606-8502, Japan. ${ }^{36}$ Department of Physics, Royal Institute of Technology (KTH), AlbaNova, SE-106 91 Stockholm, Sweden. ${ }^{37}$ Research Institute for Science and Engineering, Waseda University, 3-4-1, Okubo, Shinjuku, Tokyo 169-8555, Japan. ${ }^{38}$ CNRS, IRAP, F-31028 Toulouse cedex 4, France. ${ }^{39}$ GAHEC, Université de Toulouse, UPS-OMP, IRAP, Toulouse, France. ${ }^{40}$ Department of Astronomy, Stockholm University, SE-106 91 Stockholm, Sweden. ${ }^{41}$ sstituto Nazionale di Fisica Nucleare, Sezione di Torino, I-10125 Torino, Italy. ${ }^{42}$ Department of Physics and Department of Astronomy, University of Maryland, College Park, MD 20742, USA. ${ }^{43}$ Hiroshima Astrophysical Science Center, Hiroshima University, Higashi-Hiroshima, Hiroshima 7398526, Japan. ${ }^{44}$ Istituto Nazionale di Fisica Nucleare, Sezione di Roma "Tor Vergata," I-00133 Roma, Italy ${ }^{45}$ INTEGRAL Science Data Centre, CH-1290 Versoix, Switzerland. ${ }^{46}$ Institute of Space and Astronautical Science, JAXA, 3-1-1 Yoshinodai, Chuo-ku, Sagamihara, Kanagawa 252-5210, Japan. ${ }^{47}$ Department of Physics and Astronomy, University of Denver, Denver, CO 80208, USA ${ }^{48}$ Max-Planck-Institut für Physik, D-80805 München, Germany. ${ }^{49}$ Department of Physics and Center for Space Sciences and Technology, University of Maryland Baltimore County, Baltimore, MD 21250, USA. ${ }^{50}$ Center for Research and Exploration in Space Science and Technology (CRESST) and NASA Goddard Space Flight Center, Greenbelt, MD 20771, USA. ${ }^{51}$ Harvard-Smithsonian Center for Astrophysics, Cambridge, MA 02138, USA. ${ }^{52}$ Institut für Astro- und Teilchenphysik and Institut für Theoretische Physik, Leopold-Franzens-Universität Innsbruck, A-6020 Innsbruck, Austria. ${ }^{53}$ Department of Physics, California Polytechnic State University, San Luis Obispo, CA 93401, USA. ${ }^{54}$ Department of Physics, University of Washington, Seattle, WA 98195-1560, USA. ${ }^{55}$ Max-Planck-Institut für Kernphysik, D-69029 Heidelberg, Germany. ${ }^{56}$ Space Sciences Division, NASA Ames Research Center, Moffett Field, CA 94035-1000, USA. ${ }^{57}$ NYCB Real-Time Computing Inc. Lattingtown, NY 11560-1025, USA. ${ }^{58}$ Astronomical Observatory, Jagiellonian University, 30-244 Kraków, Poland. ${ }^{59}$ Department of Chemistry and Physics, Purdue University Calumet, Hammond, IN 46323-2094, USA. ${ }^{60}$ Institució Catalana de Recerca i Estudis Avançats (ICREA), Barcelona, Spain. ${ }^{61}$ Consorzio Interuniversitario per la Fisica Spaziale (CIFS), I-10133 Torino, Italy. ${ }^{62}$ Dipartimento di Fisica, Università di Roma "Tor Vergata," I-00133 Roma, Italy. ${ }^{63}$ Department of Physics, Center for Cosmology and Astro-Particle Physics, Ohio State University, Columbus, OH 43210, USA.

*To whom correspondence should be addressed. E-mail: majello@slac.stanford.edu (M.A.); buehler@stanford.edu (R.B.); anita.reimer@uibk.ac.at (A.R.)

†Present address: Naval Research Laboratory, Washington, DC 20375, USA.

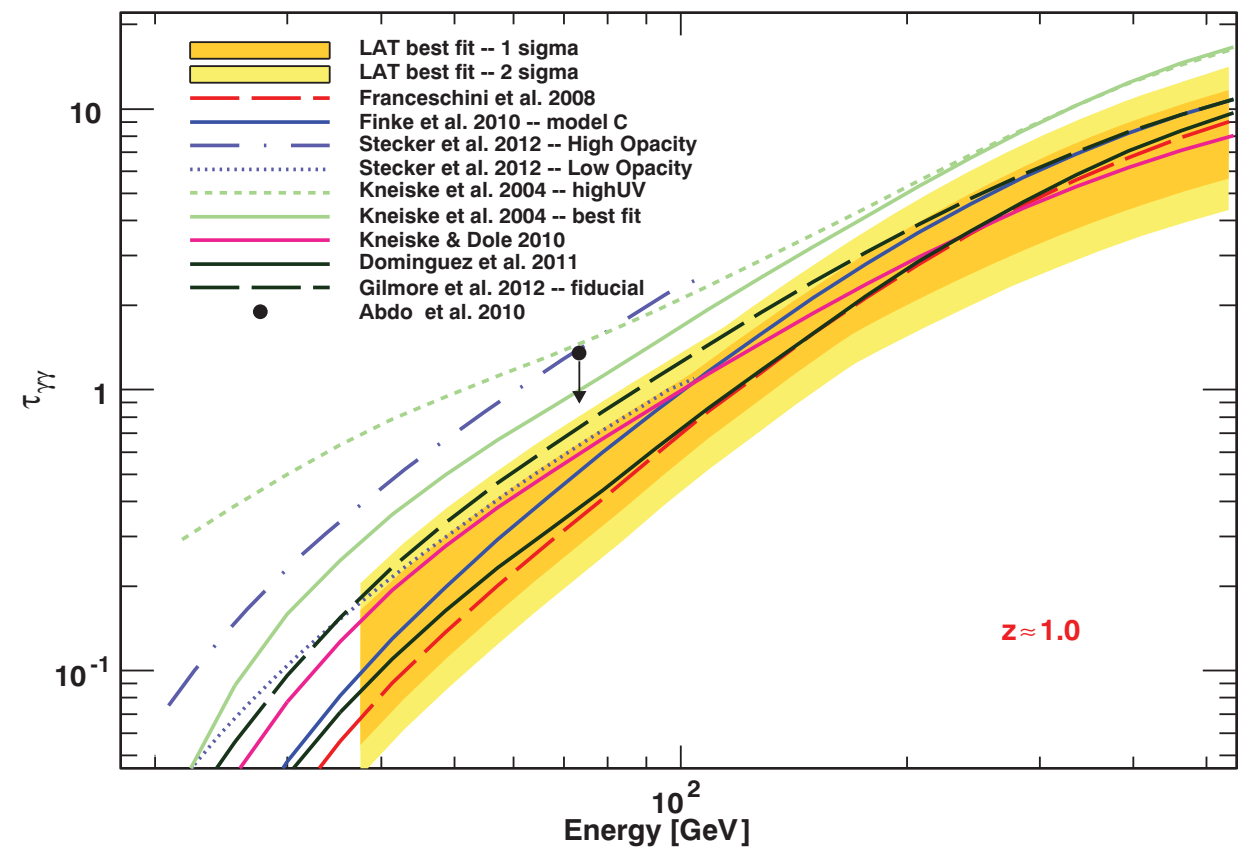




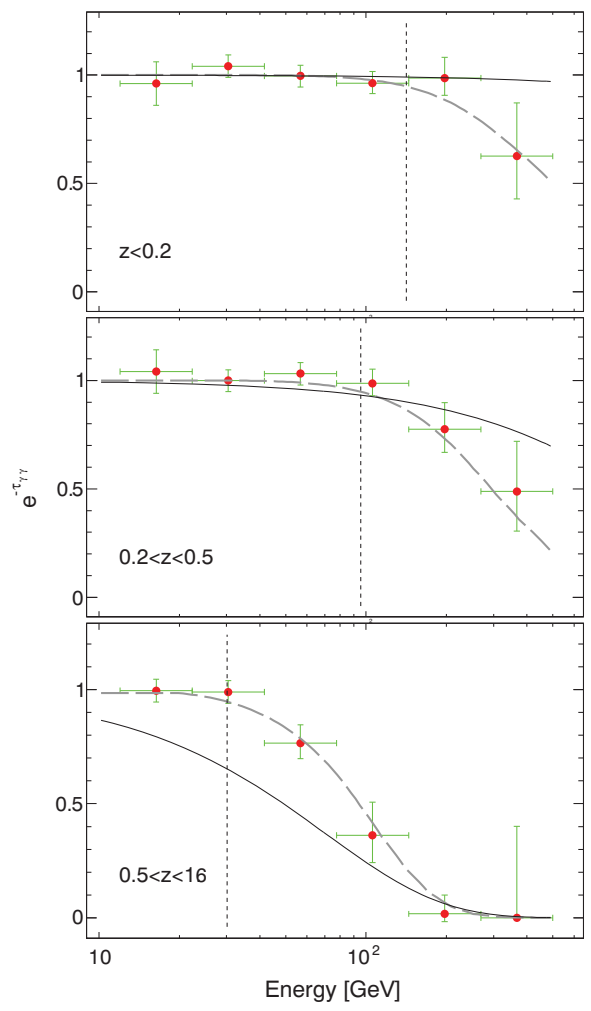

Fig. 2. Absorption feature present in the spectra of $B L$ Lac objects as a function of increasing redshift (data points, from top to bottom). The dashed curves show the attenuation expected for the sample of sources by averaging, in each redshift and energy bin, the opacities of the sample [the model of (7) was used] and multiplying this average by the best-fit scaling parameter $b$ obtained independently in each redshift interval. The vertical line shows the critical energy $E_{\text {crit }}$ below which $\leq 5 \%$ of the source photons are absorbed by the EBL. The thin solid curve represents the best-fit model, assuming that all the sources have an intrinsic exponential cutoff and that blazars follow the blazar sequence model of $(32,33)$.

apparent from Fig. 2, it appears difficult to reconcile the observed feature with an intrinsic characteristic of the blazars' spectra. We therefore associate the spectral feature to the EBL absorption.

At energies $\leq 100 \mathrm{GeV}$, gamma rays observed at Earth and coming from redshift $\geq 1$ interact mostly with UV photons of $\geq 5$ electron volts. An UV background in excess of the light emitted by resolved galaxies can be produced locally by active galactic nuclei (AGN) or at higher redshift ( $z \approx 7$ to 15 ) by low-metallicity massive stars (35). By comparing the results from the best-fit EBL models, we measured the UV component of the EBL to have an intensity of $3( \pm 1) \mathrm{nW} \mathrm{m} \mathrm{mr}^{-1}$ at $z \approx 1$. A contribution to the UV background from AGN as large as the one predicted by (36) (i.e., $\approx 10 \mathrm{nW} \mathrm{m}^{-2} \mathrm{sr}^{-1}$ ) and used in the EBL model of (22) is thus excluded by our analysis at high confidence. However, the recent prediction (37) of the UV background from AGN $\left(\approx 2 \mathrm{nW} \mathrm{m}^{-2} \mathrm{sr}^{-1}\right)$ is in agreement with the Fermi measurement. Direct measurements of the extragalactic UV background are hampered by the strong dust-scattered Galactic radiation (38). The agreement between the intensity of the UV background as measured with Fermi and that due to galaxies individually resolved by the Hubble Space Telescope (39) $\left(3 \pm 1 \mathrm{nW} \mathrm{m}^{-2} \mathrm{sr}^{-1}\right.$ versus 2.9-3.9 $\mathrm{nW} \mathrm{m} \mathrm{sr}^{-1}$, respectively) shows that the room for any residual diffuse UV emission is small. This conclusion is reinforced by the good agreement of the Fermi measurement and the estimate of the average UV background, at $z \geq 1.7$, of 2.2 to $4.0 \mathrm{nW} \mathrm{m}{ }^{-2} \mathrm{sr}^{-1}$ using the proximity effect in quasar spectra (40).

Zero-metallicity population-III stars or lowmetallicity population-II stars are thought to be the first stars to form in the universe and formally marked the end of the dark ages when, with their UV light, these objects started ionizing the intergalactic medium (41). These stars, whose mass might have exceeded 100 times the mass of our Sun $\left(M_{\odot}\right)$, are also believed to be responsible for creating the first metals and dispersing them in the intergalactic medium (42-44). A very large contribution of populationIII stars to the near-infrared EBL had already been excluded by (15). Our measurement constrains, according to $(45,46)$, the redshift of maximum formation of low-metallicity stars to be at $z \geq 10$ and its peak comoving star-formation rate to be lower than $0.5 M_{\odot} \mathrm{Mpc}^{-3}$ year $^{-1}$. This upper limit is already of the same order of the peak star-formation rate of 0.2 to $0.6 M_{\odot} \mathrm{Mpc}^{-3}$ year $^{-1}$ proposed by (47) and suggests that the peak star-formation rate might be much lower, as proposed by (48).

\section{References and Notes}

1. X. Fan et al., Astron. J. 132, 117 (2006).

2. R. C. Gilmore, P. Madau, J. R. Primack, R. S. Somerville, F. Haardt, Mon. Not. R. Astron. Soc. 399, 1694 (2009).

3. S. Inoue et al., Mon. Not. R. Astron. Soc. 404, 1938 (2010); http://arxiv.org/pdf/1007.4379.pdf.

4. R. J. Gould, G. Schréder, Phys. Rev. Lett. 16, 252 (1966).

5. G. G. Fazio, F. W. Stecker, Nature 226, 135 (1970).

6. F. W. Stecker, O. C. de Jager, M. H. Salamon, Astrophys. J. 390, L49 (1992)

7. A. Franceschini, G. Rodighiero, M. Vaccari, Astron Astrophys. 487, 837 (2008).

8. We define the critical energy $E_{\text {crit }}$ such that less than $5 \%$ of the source photons are absorbed for the EBL model of (7) below this energy.

9. D. H. Hartmann, in The First GLAST Symposium, S. Ritz, P. Michelson, C. A. Meegan, Eds. (2007), vol. 921 of American Institute of Physics Conference Series, pp. 24-25.

10. F. W. Stecker, in The First GLAST Symposium, S. Ritz, P. Michelson, C. A. Meegan, Eds. (2007), vol. 921 of American Institute of Physics Conference Series, pp. 237-239.

11. A. Kashlinsky, D. Band, in The First GLAST Symposium, S. Ritz, P. Michelson, C. A. Meegan, Eds. (2007), vol. 921 of American Institute of Physics Conference Series, pp. 243-245.

12. K. Mannheim, S. Westerhoff, H. Meyer, H. H. Fink, Astrophys. J. 315, 77 (1996); http://arxiv.org/pdf/ astro-ph/9703184.pdf.

13. A. A. Abdo et al., Astrophys. J. 723, 1082 (2010).

14. M. Raue, Astron. Astrophys. 520, A34 (2010).

15. F. Aharonian et al., Nature 440, 1018 (2006).

16. M. Ackermann et al., Astrophys. J. 743, 171 (2011).

17. A. Reimer, Astrophys. J. 665, 1023 (2007).

18. A more detailed description is given in the supplementary materials on Science Online.

19. A. Rau et al., Astron. Astrophys. 538, A26 (2012).

20. The software to perform analysis of Fermi data is available at http://fermi.gsfc.nasa.gov/ss/data/analysis/software/.

21. P. Nolan et al., arXiv:1108.1435 (2011).

22. T. M. Kneiske, T. Bretz, K. Mannheim, D. H. Hartmann, Astron. Astrophys. 413, 807 (2004).
23. F. W. Stecker, M. A. Malkan, S. T. Scully, Astrophys. J. 648, 774 (2006)

24. J. D. Finke, S. Razzaque, C. D. Dermer, Astrophys. J. 712, 238 (2010).

25. A. Domínguez et al., Mon. Not. R. Astron. Soc. $\mathbf{4 1 0}$ 2556 (2011).

26. T. M. Kneiske, H. Dole, Astron. Astrophys. 515, A19 (2010).

27. R. C. Gilmore, R. S. Somerville, ]. R. Primack, A. Domínguez, Mon. Not. R. Astron. Soc. 422, 3189 (2012).

28. D. Mazin, M. Raue, Astron. Astrophys. 471, 439 (2007).

29. The MAGIC Collaboration et al., Science 320, 1752 (2008)

30. We show in the supplementary materials that our result is robust against conservative choices of the critical energy.

31. G. Ghisellini, A. Celotti, G. Fossati, L. Maraschi, A. Comastri, Mon. Not. R. Astron. Soc. 301, 451 (1998).

32. G. Fossati, L. Maraschi, A. Celotti, A. Comastri, G. Ghisellini, Mon. Not. R. Astron. Soc. 299, 433 (1998).

33. G. Ghisellini, L. Maraschi, F. Tavecchio, Mon. Not. R. Astron. Soc. 396, L105 (2009).

34. E. T. Meyer, G. Fossati, M. Georganopoulos, M. L. Lister, Astrophys. J. 740, 98 (2011).

35. M. R. Santos, V. Bromm, M. Kamionkowski, Mon. Not. R. Astron. Soc. 336, 1082 (2002).

36. F. Haardt, P. Madau, Astrophys. J. 461, 20 (1996).

37. F. Haardt, P. Madau, Astrophys. J. 746, 125 (2012).

38. S. Bowyer, Annu. Rev. Astron. Astrophys. 29, 59 (1991)

39. J. P. Gardner, T. M. Brown, H. C. Ferguson, Astrophys. J. 542, L79 (2000).

40. J. Scott, ]. Bechtold, A. Dobrzycki, V. P. Kulkarni, Astrophys. J. 130, 67 (2000)

41. V. Bromm, R. B. Larson, Annu. Rev. Astron. Astrophys. 42, 79 (2004)

42. J. P. Ostriker, N. Y. Gnedin, Astrophys. J. 472, L63 (1996).

43. T. H. Greif, J. L. Johnson, V. Bromm, R. S. Klessen, Astrophys. J. 670, 1 (2007).

44. J. H. Wise, T. Abel, Astrophys. J. 685, 40 (2008).

45. M. Raue, T. Kneiske, D. Mazin, Astron. Astrophys. 498, 25 (2009).

46. R. C. Gilmore, arXiv:1109.0592 (2011).

47. V. Bromm, A. Loeb, Astrophys. J. 575, 111 (2002).

48. L. Tornatore, A. Ferrara, R. Schneider, Mon. Not. R. Astron. Soc. 382, 945 (2007).

49. F. W. Stecker, M. A. Malkan, S. T. Scully, arXiv:1205.5168 (2012).

Acknowledgments: M.A. acknowledges generous support from the Fermi guest investigator program (proposals ID 31117 and 51258) and the Swift and the Gamma-Ray Burst Optical/ Near-Infrared Detector (GROND) teams for observing 100 Fermi blazars in an effort to constrain their redshifts. The Fermi LAT Collaboration acknowledges generous ongoing support from a number of agencies and institutes that have supported both the development and the operation of the LAT as well as scientific data analysis. These include the National Aeronautics and Space Administration and the Department of Energy in the United States; the Commissariat à l'Energie Atomique and the Centre National de la Recherche Scientifique/Institut National de Physique Nucléaire et de Physique des Particules in France; the Agenzia Spaziale Italiana and the Istituto Nazionale di Fisica Nucleare in Italy; the Ministry of Education, Culture, Sports, Science and Technology (MEXT), High Energy Accelerator Research Organization (KEK), and Japan Aerospace Exploration Agency (JAXA) in Japan; and the K. A. Wallenberg Foundation, the Swedish Research Council, and the Swedish National Space Board in Sweden. Additional support for science analysis during the operations phase is gratefully acknowledged from the Istituto Nazionale di Astrofisica in Italy and the Centre National d'Etudes Spatiales in France. J. Conrad is funded by a grant from the K. A. Wallenberg Foundation. E. Troja is a NASA Postdoctoral Program Fellow.

\section{Supplementary Materials}

www.sciencemag.org/cgi/content/full/science.1227160/DC1 Materials and Methods

Supplementary Text

Figs. S1 to S4

Table S1

References (50-68)

9 July 2012; accepted 11 October 2012

Published online 1 November 2012;

10.1126/science. 1227160 